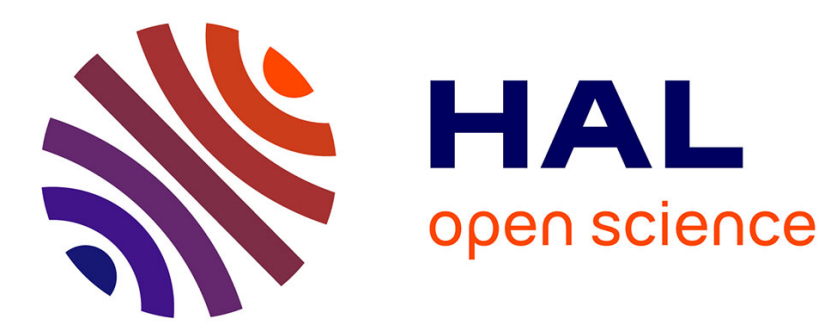

\title{
Emergence of structured interactions: from a theoretical model to pragmatic robotics
}

\author{
Arnaud Revel, Pierre Andry
}

\section{To cite this version:}

Arnaud Revel, Pierre Andry. Emergence of structured interactions: from a theoretical model to pragmatic robotics. Neural Networks, 2009, 22 (2), pp.116-125. hal-00417847

\section{HAL Id: hal-00417847 \\ https://hal.science/hal-00417847}

Submitted on 17 Sep 2010

HAL is a multi-disciplinary open access archive for the deposit and dissemination of scientific research documents, whether they are published or not. The documents may come from teaching and research institutions in France or abroad, or from public or private research centers.
L'archive ouverte pluridisciplinaire HAL, est destinée au dépôt et à la diffusion de documents scientifiques de niveau recherche, publiés ou non, émanant des établissements d'enseignement et de recherche français ou étrangers, des laboratoires publics ou privés. 


\title{
Emergence of structured interactions : from a theoretical model to pragmatic robotics
}

\author{
A.Revel ${ }^{1}$, P.Andry ${ }^{2}$ \\ ETIS, CNRS UMR 8051, ENSEA, Univ Cergy-Pontoise, F-95000 Cergy-Pontoise, France
}

\begin{abstract}
In this article, we present two neural architectures for the control of socially interacting robots. Beginning with a theoretical model of interaction inspired by developmental psychology, biology and physics, we present two subcases of the model that can be interpreted as "turn-taking" and "synchrony" at the behavioral level. These neural architectures are both detailed and tested in simulation. A robotic experiment is even presented for the "turn-taking" case. We then discuss the interest of such behaviors for the development of further social abilities in robots.
\end{abstract}

Key words: Robotics, Social Interaction, Neural models, Development

\section{Introduction}

With the democratisation of robotics, an important effort is made to make those "technological" machines more attractive for human beings. For instance, a major focus of interest is put on the expressiveness and the aspect of the robots [112734: the question is then how to design "cute" robots? Nevertheless, if the aim of these approaches is to facilitate human-machine communication, they often neglect to consider the dynamics of the interaction between two agents. In our opinion, intuitive communication (verbal or not) refers to the ability "to take turn" in the interaction or to be "synchronized" with the other: in summary, to adapt its own dynamics to the other's behavior via the integration of the global dynamical exchange. In consequence, our aim is to design robotic controllers that can adapt to the interaction, embed fundamental dynamical properties, and make emerge communicative behaviors such as the "ability to synchronize" and "alter-

1 E-mail: revel@ensea.fr

2 E-mail: andry@ensea.fr

Preprint submitted to Neural Networks nate the exchange". To support this approach, we take inspiration from different scientific fields that have to deal with interaction: dynamical systems, developmental psychology, and neurobiology.

In this paper, we will show how "synchrony" and "alternation" can emerge from a simple neural model of interaction. Next, the underlying dynamical parameters of the model will be applied to 2 robotic controllers in order to increase their interactional abilities.

\section{Neural model of agents interaction}

\subsection{Inspiration}

\subsubsection{Developmental psychology}

Developmental psychology aims at understanding how the sensory, motor and cognitive capabilities of the individual evolve from birth. In the frame of longitudinal studies applied to populations with selected developmental ages, researches detect and observe the emergence, or the disappearance of behaviors, allowing to formulate hypothesis and models of 
the underlying sensori-motor and cognitive mechanisms. In this discipline, a lot of issues are concerned with the progressive rise of communication among babies and young children.

It has been described that human communication can adopt several modalities [7]: language, paralanguage and kinesic. Paralanguage are the nonverbal voice and sounds which can be emitted. Kinesic stands for the "body-language" (facial expressions, gaze, gestures, postures, head and body movements, haptics and proxemics). Obviously, nonverbal communication is anterior to language in development.

As our goal is to tackle intuitive communication, and as our robots are not verbal, we are essentially interested in that latter kind of communication.

At a behavioral point of view, it has been shown that in our daily activities the social context is formatted by physical interactions. One example is, for instance, the emergence of rhythmic applause in a crowd 38]. Another interesting example is the fact that we can adopt a similar posture 9] when interacting with another. More precociously, adults and neonate participate in a social interaction via head movements 43 what seems to suggest that very early in development, humans are equipped to deal with social interaction.

In particular, Neo-natal imitation (from 323329 ), has been widely studied for its presumed implication in communication. In neo-natal imitation the observation by the baby of facial expressions performed by the experimenter gives rise to basic imitation (tongue protrusion, eye blinking, vocalizations). As experiments has been done with babies aged of a few minutes old, this kind of imitation is not supposed to be learned. This phenomenon asks an important question concerning the function of such a low-level imitative behavior. Answering this question, a few developmental psychologists have emphasized that imitation has not only a learning function but also a communication one 3612. They have suggested that neo-natal imitation is a pre-linguistic mode of communication that does not require any interaction protocol but can be the basis of subsequent higher level communication and social abilities. More precisely, Nadel has emphasized the fact that synchrony and "turn-taking" are fundamental for communication [37].

Several recent experiments seem to validate this point of view. Oullier, for instance 41, has proposed a simple experimental paradigm in which pairs of participants facing each other are required to ac- tively produce actions, while provided (or not) with the vision of similar actions being performed by someone else. Results reveal that spontaneous phase synchrony (i.e. in-phase co-ordinated behavior) between two people emerges as soon as they exchange visual information, even if they are not explicitly instructed to co-ordinate with each other.

In a similar experiment, 26] tries to identify whether an interpersonal motor co-ordination emerges between two participants when they intentionally tried to not co-ordinate their movements between each other. The goal of the first two situations was for participants to intentionally co-ordinate or not co-ordinate their movements between each other. The results revealed in the "not co-ordinate" condition the emergence of an unintended co-ordination in the frequency domain. What is however interesting to notice is that the results also reveal the presence of individual intrinsic motor properties (motor signature) in the 2 conditions. These results indicate that, when there was information sharing, participants could not avoid (unintentionally) co-ordinating with someone.

This is consistent with the results found by [55] in EEG. In a specially designed dual electroencephalogram system, pairs of participants executing self-paced rhythmic finger movements with and without vision of each other's actions were continuously monitored and recorded. After analysis, a pair of oscillatory components (phi1 and phi2) located above the right centro-parietal cortex distinguished effective from ineffective co-ordination: increase of phi1 favored independent behavior and increase of phi2 favored co-ordinated behavior. The author's hypothesis is that the phi complex rejects the influence of the other on a person's ongoing behavior, with phi1 expressing the inhibition of the human mirror neuron system and phi2 its enhancement.

In this section, we have shown that synchronization behaviors exist very early in human and persists in time. This can suggest that they could have a early and perennial effect on the structure of interpersonal non-verbal communication.

\subsubsection{Synchrony in biological systems}

We have seen that behavioral synchronisation exists in social contexts. The question is then: are they individual mechanisms that can help synchrony between humans?

In fact, many biological activities in human are naturally synchronized. For instance, it has been 
shown that heartbeat, respiration and locomotion in humans walking or running on a treadmill are synchronized 40.

The role of circadian rhythm has also been proved to have effect on the oscillation of hormone secretion, core body temperature and lymphocyte number 33548 .

It has also been shown that we are well equipped to satisfy auto-synchrony (for instance with fingers 22 ). What is interesting to note is that the result is different in function of speed of motion: we are in phase for slow motion, and anti-phase for increasing speed.

When regarding underlying neural circuits, there is also evidence that synchrony exists within brain areas and between them, and is even crucial for information processing 5649 . For instance, synchronization in the visual cortex seems responsible of binding of related visual features [20 56]. Synchronization of oscillatory activity in the sensorimotor cortex serve also for the integration and coordination of information underlying motor control 31. Simultaneous spiking in neural population has also been observed in olfaction [51] and touch [50].

In certain cases, synchronization of neural populations leads to pathological disorders. For instance, Parkinson tremors seem to be due to synchronization between the cortex and the basal ganglia 1554 .

\subsubsection{Dynamical systems}

In 1665, C.Huygens described how two clocks anti-synchronize after a while by mutually interacting together 23. Since this discovery, the selforganization of synchronization phenomenon has been widely studied by nonlinear dynamics 2139 . In physics, relaxation oscillators have been modeled by Van Der Pol 46. More recently, the synchronization mechanisms has been deeply studied between 2 or several oscillators, even in the case of chaotic systems 454253 . This has lead to a better understanding and modeling of the mechanisms involved during mutual interaction.

As far as we are concerned, this discipline provides a theoretical framework for the study of interacting/coupled social systems.

Taking inspiration from non-linear dynamics we propose that basic dynamical social properties may emerge from the dynamical perception/action interaction of two identical oscillatory systems in interaction. The main idea is that the value of the oscil- lators embedded in each robot models an internal propensity to "interact" with the other robot.

The oscillator is supposed to be connected both to sensations and actions: perception modulates this oscillator while this latter modifies the actions (inhibition, modulation, activation...).

\subsection{Formal model of the oscillator}

The oscillator we use is made of 2 neurons $(u$ and $v$ ) inhibiting each other proportionally to the parameter $\beta$. The equations are given in 1 (see also fig (1).

$\left\{\begin{array}{l}u(n+1)=f(u(n)-\beta \cdot v(n)) \\ v(n+1)=f(v(n)+\beta \cdot u(n))\end{array}\right.$

With $f($.$) being the activating function of the neu-$ rons.

In a first approximation, we consider the function $f($.$) as the identity function. Equation 1 becomes:$

$\left\{\begin{array}{l}u(n+1)=u(n)-\beta \cdot v(n) \\ v(n+1)=v(n)+\beta \cdot u(n)\end{array}\right.$

By approximating the derivative of a function by: $f^{\prime}(x+1) \simeq f(x+1)-f(x)$. Equation 2 turns into:

$\left\{\begin{array}{l}u^{\prime}(t)=-\beta \cdot v(t) \\ v^{\prime}(t)=\beta \cdot u(t)\end{array}\right.$

By deriving the first line of equation and simplifying equation 3 we get:

$$
u^{\prime \prime}(t)+\beta^{2} \cdot u(t)=0
$$

Solutions of such an equation have the following form:

$$
u(t)=A \cdot e^{i \beta t}+c n t
$$

That is an oscillatory function with frequency $\nu=$ $\frac{\beta}{2 \pi}$

\subsubsection{Linking 2 oscillators together}

When considering the interaction between the 2 agents, it can simply be obtained by linking the output of neuron $u_{1}$ of agent 1 (considered as the "action" performed by agent 1) to the input of neuron $u_{2}$ of agent 2 (considered as the "sensation" of agent 2) and reversely.

In that case, we want to study the form of the interaction between the 2 systems. 


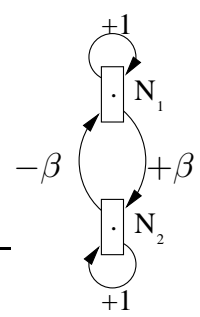

Fig. 1. The oscillator is made of 2 neurons, $u$ and $v$, with a self-connection weighted to 1 . A link whose weight is $+\beta$ connects $v$ to $u$, and a link whose weight is $-\beta$ connects $u$ to $v$.

Formally, this interaction can be modeled by transforming equation 3 into:

$$
\left\{\begin{array}{l}
u_{1 / 2}^{\prime}(t)=-\beta_{1 / 2} \cdot v_{1 / 2}(t)+I_{21 / 12} \cdot u_{2 / 1}(t) \\
v_{1 / 2}^{\prime}(t)=\beta_{1 / 2} \cdot u_{1 / 2}(t)
\end{array}\right.
$$

With $I_{21}$ the value of the inhibition between agent 2 and 1 and $I_{12}$ the value of the inhibition between agent 1 and 2 .

By deriving the first line of those equations and by reducing with the second line, we get:

$$
\left\{\begin{array}{l}
u_{1}^{\prime \prime}(t)=-\beta_{1}^{2} \cdot u_{1}(t)+I_{21} \cdot u_{2}^{\prime}(t) \\
u_{2}^{\prime \prime}(t)=-\beta_{2}^{2} \cdot u_{2}(t)+I_{12} \cdot u_{1}^{\prime}(t)
\end{array}\right.
$$

Let us consider to simplify that: $I_{12}=I_{21}=\varepsilon$ a weak value of the interaction. The previous equation becomes:

$$
\left\{\begin{array}{l}
u_{1}^{\prime \prime}(t)=-\beta_{1}^{2} \cdot u_{1}(t)+\varepsilon \cdot u_{2}^{\prime}(t) \\
u_{2}^{\prime \prime}(t)=-\beta_{2}^{2} \cdot u_{2}(t)+\varepsilon \cdot u_{1}^{\prime}(t)
\end{array}\right.
$$

2.2.1.1. Oscillatory solutions We consider here that oscillatory solutions can exists. For that purpose, we consider the variables $\left(A_{1}\right.$ and $A_{2}$ being complex):

$$
\begin{cases}x_{1}=u_{1}=A_{1} e^{i \omega t} & x_{2}=u_{2}=A_{2} e^{i \omega t} \\ y_{1}=u_{1}^{\prime}=i \omega A_{1} e^{i \omega t} & y_{2}=u_{2}^{\prime}=i \omega A_{2} e^{i \omega t}\end{cases}
$$

Considering that 7 can be rewritten as:

$$
\left\{\begin{array}{l}
u_{1}^{\prime \prime}(t)-\omega^{2}=\left(\omega^{2}-\beta_{1}^{2}\right) \cdot u_{1}(t)+\varepsilon \cdot u_{2}^{\prime}(t) \\
u_{2}^{\prime \prime}(t)-\omega^{2}=\left(\omega^{2}-\beta_{2}^{2}\right) \cdot u_{2}(t)+\varepsilon \cdot u_{1}^{\prime}(t)
\end{array}\right.
$$

We can deduce that:

$$
\left\{\begin{array}{l}
A_{1}^{\prime}(t)=-i \cdot\left(\frac{\left(\omega^{2}-\beta_{1}^{2}\right)}{\omega}\right) \cdot A_{1}(t)+\varepsilon \cdot A_{2} \\
A_{2}^{\prime}(t)=-i \cdot\left(\frac{\left(\omega^{2}-\beta_{2}^{2}\right)}{\omega}\right) \cdot A_{2}(t)+\varepsilon \cdot A_{1}
\end{array}\right.
$$

As $\omega \simeq \beta_{1} \simeq \beta_{2}, \frac{\left(\omega^{2}-\beta^{2}\right)}{\omega}=\frac{(\omega-\beta)(\omega+\beta)}{\omega} \simeq 2 \cdot(\omega-$ $\beta)$. If we set $\omega=\frac{\beta_{1}+\beta_{2}}{2}$ and $\chi=\frac{\beta_{1}^{\omega}-\beta_{2}}{2}$, equation 9] becomes:

$$
\left\{\begin{array}{l}
A_{1}^{\prime}(t)=2 i \chi A_{1}+\varepsilon \cdot A_{2} \\
A_{2}^{\prime}(t)=-2 i \chi A_{2}+\varepsilon \cdot A_{1}
\end{array}\right.
$$

As $A$ is a complex amplitude, we can turn it into $A=R \cdot e^{i \Phi}$.

After substitution in 10 and simplifications, we get:

$$
\left\{\begin{array}{l}
R_{1}^{\prime}(t)+R_{1} i \Phi_{1}^{\prime}=2 i \chi R_{1}+\varepsilon \cdot R_{2} e^{i\left(\Phi_{2}-\Phi_{1}\right)} \\
R_{2}^{\prime}(t)+R_{2} i \Phi_{2}^{\prime}=2 i \chi R_{2}+\varepsilon \cdot R_{1} e^{i\left(\Phi_{1}-\Phi_{2}\right)}
\end{array}\right.
$$

Grouping real and imaginary terms and considering $\Psi=\Phi_{2}-\Phi_{1}$ leads to:

$\left\{\begin{array}{l}R_{1}^{\prime}(t)=\varepsilon R_{2} \cos \Psi \quad \Phi_{1}^{\prime}=2 \chi+\varepsilon \cdot \frac{R_{2}}{R_{1}} \sin \Psi \\ R_{2}^{\prime}(t)=\varepsilon R_{1} \cos \Psi \quad \Phi_{2}^{\prime}=2 \chi-\varepsilon \cdot \frac{R_{1}}{R_{2}} \sin \Psi\end{array}\right.$

As $\Psi^{\prime}=\Phi_{2}^{\prime}-\Phi_{1}^{\prime}$, we finally reach the following equation:

$$
\left\{\begin{array}{rlrl}
R_{1}^{\prime}(t) & = & & \varepsilon R_{2} \cos \Psi \\
R_{2}^{\prime}(t) & = & & \varepsilon R_{1} \cos \Psi \\
\Psi^{\prime} & =-\varepsilon \cdot\left(\frac{R_{2}}{R_{1}}-\frac{R_{1}}{R_{2}}\right) \sin \Psi
\end{array}\right.
$$

The equation in $\Psi$ has different behavior according to the sign of $\varepsilon$.

2.2.1.2. If $\varepsilon>0$ (positive coupling) The stable solution stands for $\Psi=0=$ "synchronous case".

Indeed, in that case, supposing that $\frac{R_{2}}{R_{1}}-\frac{R_{1}}{R_{2}}=\frac{1}{\tau}$ :

$\Psi^{\prime} \simeq-\varepsilon \Psi$

Whose solution is $\Psi=e^{-\frac{\varepsilon}{\tau} t}$.

The only stable solution stands for $\varepsilon>0$.

2.2.1.3. If $\varepsilon<0$ (negative coupling) The stable solution stands for $\Psi=\pi=$ "phase opposition".

Indeed, in that case, supposing that $\Psi=\pi+\phi$ :

$\Psi^{\prime}=\phi^{\prime}=-\varepsilon \sin (\pi+\phi)=\varepsilon \sin \phi \simeq \varepsilon \phi$

Whose solution is $\phi=e^{\frac{\varepsilon}{\tau} t}$ with a stable solution only if $\varepsilon<0$. 


\subsubsection{Interpretation of the model for social} interaction

Given this theoretical framework, we can give an interpretation of this model at the level of social interaction. The oscillator could be an abstraction of the agent's motivation for action: if the oscillator is "up", the system tends to produce actions; if the oscillator is "down", it does not produce actions. The inhibition coupling is then a model of the interaction between the agents which is supposed to be "perfect" (without loose of energy).

2.2.2.1. Phase opposition $(\varepsilon<0)$ In this case, we are in the situation where the two oscillators reciprocally alternate in motor production: when an agent acts the other stops any action until this situation alternates. It is interesting to notice that in such a case, the alternation itself is an emergent property of coupling. Interpreting this situation as a social interaction, the model suggests that a "turn-taking" behaviour, as demonstrated by young children, may naturally emerge from the dynamical interaction between 2 agents (cf. section 2.1.1).

2.2.2.2. Phase synchronisation $(\varepsilon>0)$ In this case, the interaction allows to reciprocally exchange energy which results in the synchronization of oscillators: both agents performs the same action at the same time. It typically corresponds to a minimal interaction, where each agent is able to produce its motor repertoire, and at the same time to modulate its production in order to adapt to the other's one. In other words, solving the trade-off between producing actions of self and observing those of others, as observed among young children naturally performing immediate and spontaneous imitations in the frame of pre-verbal communication (cf. section 2.1.1).

In the following section, we will consider those two sub-cases in the context of robotics control architectures.

\section{Designing an interactive robotic controller}

\subsection{The case of negative coupling: turn taking}

In this section, we explore the idea that the formal model developed above can make a kind of "turntaking" behaviour emerge from the dynamical interaction between 2 agents (see figure 2).

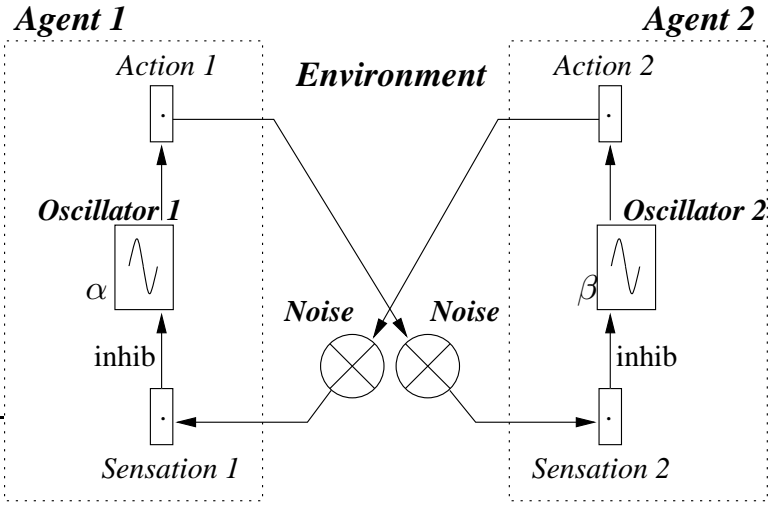

Fig. 2. Architecture of the two agents influencing each other. Each agent is driven by an internal oscillator and produces actions depending on this oscillator. A noise due to the environment and the hardware devices appears on the signal between the two oscillators.

\subsubsection{Simulation results}

To test the conditions and the limits of the antisynchronization between the two oscillators in interaction we varied the different parameters in simulation: the oscillator 1 was kept unchanged $\left(\beta_{1}=0.2\right)$ while the oscillator 2 was varied between 0.02 and 0.4 with a step of 0.01 . The inhibition between the two systems was also varied between 0 . and 0.1 with a step of 0.02. We observe in figure 3 Left. which plots the difference of frequencies $f_{1}-f_{2}$ that they get completely synchronized $\left(\Delta_{f}=0\right)$ in the vicinity of the mean frequency (this is called "frequency locking"). In addition, as the inhibition increases, the locking region gets wider and wider. The regions of synchronization observed in simulation are analogous to the "phase locking domain" described as "tongues" by Arnold: when two periodic oscillators are coupled together there are parameter regions called "Arnold tongues" where they mode lock and their motion is periodic with a common frequency 2 .

Concurrently with "frequency locking", it can be observed (an example is given in figure 3 right, which plots $u_{1}(n)$ in function of $u_{2}(n)$ with $f_{1}=f_{2}$ and $I=0.5)$ that both oscillators converge to a constant phase shift of $\pi$. That precisely corresponds to anti-synchronization. This phenomenon is described as "phase locking of periodic oscillators" 52 .

\subsubsection{Robotic implementation}

To transpose the conceptual model to a robotics platform, the way the oscillators and their interaction could be embodied in a concrete robot should be considered. In particular, the reciprocal influ- 

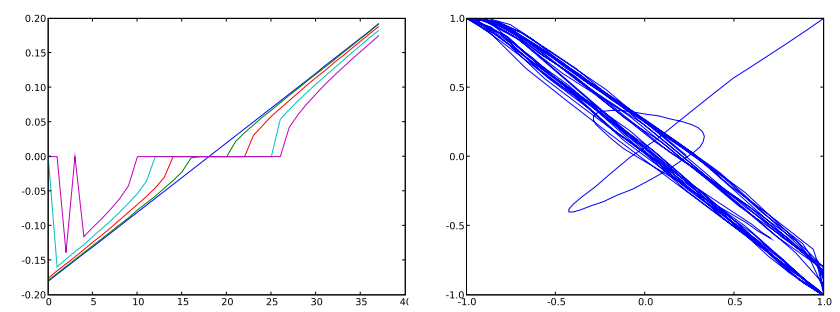

Fig. 3. a) Difference of frequencies $f_{1}-f_{2}$ for oscillators 1 and 2 in function of $f_{2}\left(f_{2} \in[0.01,0.4]\right)$ and $I$ the mutual inhibition $(I \in[0,0.1])$ between oscillators $\left(f_{1}\right.$ is fixed to $0.2)$. b) Lissajou plotting of $u_{1}(n)$ in function of $u_{2}(n)$ for the first 500 steps of the simulation.

ence between the two oscillators is then mediated by the environment. In the robotic platform ADRIANA (ADaptable Robotics for Interaction ANAlysis - see [28] for details), dedicated to the study of interaction and communication features, the detection of movements and a moving arm are used to interact (see fig 4 ).

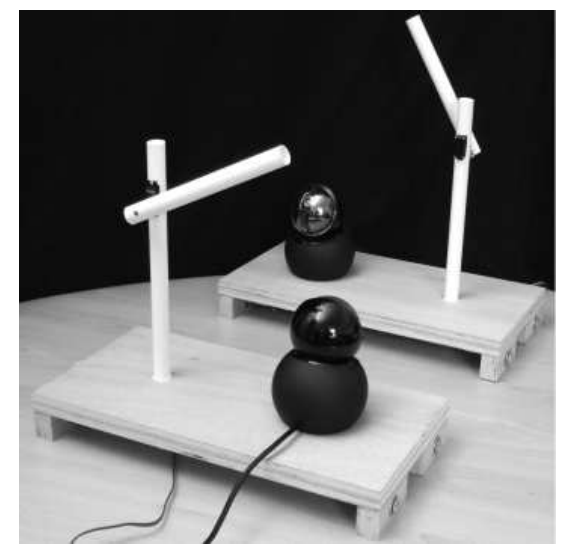

Fig. 4. In the present experiment ADRIANA is customized with a single arm for each robot and a webcam which enables the robot to see the other robot but not to see its own arm. The two robots move their arms according to their own dynamic. They influence each other by seeing the movement of the other robot.

Each robot has one arm and the camera is put in front of the arm so as each robot faces the other robot in order to perceive its arm movements. The architecture controlling each robot is made of two parts: the oscillator described in the previous section which controls the arm movements ; the image processing system which computes the inhibitory signal. The frequency of the arm movements directly depends on the activation of the oscillator: the higher the activation of $u_{1}$ is, the more frequent the arm movements are. The vision system allows to detect the motor actions of the other agent.

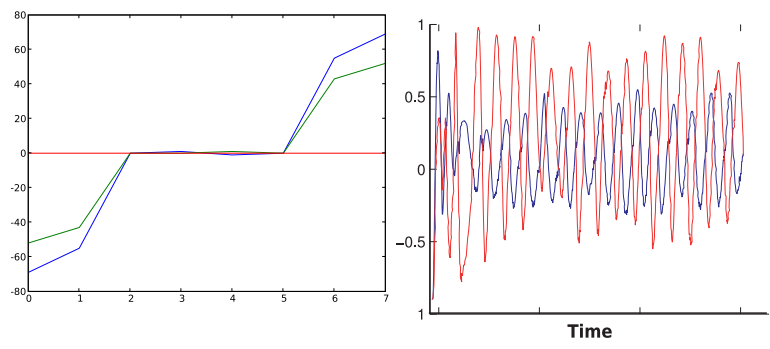

Fig. 5. Left : Difference of frequencies in function of the reciprocal inhibition. Right : Time plot of agent1 and agent2 activities. Both internal oscillators rapidly anti-synchronize thanks to the perception/action interaction.

We have first tested this architecture and setup with two robots having the same oscillator's parameter $\left(\beta_{1}=\beta_{2}\right)$. After a transition period, the two behaviors of the two robots stabilise in antisynchronization: when one robot moves the other stops and conversely, the two robots take turns. This is shown by figure 5 Left. where both internal oscillators rapidly anti-synchronize thanks to the perception/action interaction (this plot is equivalent to the Lissajou representation mention above, along the time axis).

As in simulation, we have tested the interaction between the 2 agents for several values of $f_{2}$ and I. 32 experiments were conducted with the robots in order to test the following set of parameters : the frequency ratio $\frac{\beta_{2}}{\beta_{1}}$ took the values $1,1.2,1.4$, $1.6,1.8,2,4$ and 6 , (only $\beta_{2}$ varies) the reciprocal inhibition $I$ took the values $0,0.1,0.3$ and 0.5 . The difference of frequencies is plotted in figure 5 -right. As in simulation, we can see wide region of frequency locking which varies with $I$.

\subsubsection{Summary}

This emerging dynamics have been shown to tolerate a wide range of parameters. This robustness allows the 2 systems to remain in interaction even if the conditions are not ideal: the agent's dynamics and environment may be modified along time. It suggests that the emergence of turn taking between two systems is a very robust dynamical attractor that can be trusted in order to develop further social and communicative functionalities.

This view fits physics results on chaotic oscillators which support the idea that the more chaotic oscil- 
lators are synchronized, the more information they exchange $([7])$.

\subsection{The case of positive coupling: synchrony}

In the previous section, we have seen the effects of adding an oscillator, as a component of the architecture, that modulates the perceptions and the action of the agents. In the previous robotic experiment, the perceptions and the actions of the architecture are limited to a coding of the perception (amount of movement perceived) and of the action (speed of the movement of the arm) expressed in one dimension. Nevertheless, if we take into account more elaborated control architectures such as the one that apply to complex robotic systems, it can appear that adding explicitly one internal oscillator is not so simple, because the information flow is of higher dimension, and because these systems are often able to switch from a particular dynamics to another (for example systems that have a particular dynamics during a learning phase, and a second one during the reproduction phase).

But interestingly, the dynamics of such systems is often already rich enough to provide oscillating signal without the use of any explicit ad-hoc oscillators. Traditional works on motor control have for example shown the benefit of using oscillating primitives as the basis of the building of more complex behaviors 825241310 . In previous works, we have proposed a NN architecture able to learn, predict and reproduce on-line one simple sequence of sensorimotor events. Such a system has been used in navigation (from [5] to 19]), planning 414, imitation with an eye-arm system [1730, with different variations of the NN model used to categorize and predict the sensori-motor flow.

On these systems, the behavior (i.e the sequence of sensori-motor actions) learned was most of the time cyclic, in order to obtain a system that provides continuous demonstration of the learned sequence. For example a system that learned the sequence ABCD (each letter symbolizing a different sensory-motor attractor) associated the transitions A-B, B-C, C-D, $\mathrm{D}-\mathrm{A}$, in order to reproduce cyclically the sequence ABCD. As a result, after an on-line learning period, and once they are able to predict a sensory-motor event, our robots start to reproduce it in loop, driven by their step by step predictions [181]. This provides systems that start to reproduce the behavior they have learned, once they are able to predict a sequence. Of course, a reliable demonstration of the learned sequence requires that no input is given concurrently to the system (the experimenter, or the learner stops stimulating the robot), otherwise the system will be confused between selecting an action corresponding to its own prediction (the response to its own predictions) or to its inputs (the response to the other's stimulations).

Agent 1

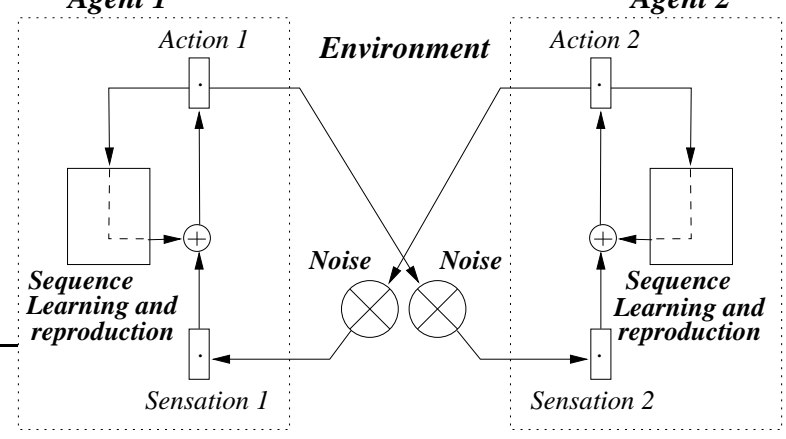

Fig. 6. Architecture of the two agents influencing each other. Each agent is now able to learn and reproduce a sequence of actions. This "learn and reproduce" mechanism induces an internal dynamics that will drive the behavior of each agent. If we suppose that an agent learns a cyclic sequence (for example ABCDA), it will then reproduce the sequence in loop, and the looping of the prediction of the sensori-motor transitions will act as an endogenous multi-stable oscillator. Interestingly, the first property that emerges from the interaction of 2 systems is the synchronization of the sensori-motor behaviors.

As a result, one could qualify such an architecture as "blind" when reproducing the sequence : in order to obtain a correct reproduction, the system executes its predictions without "listening" to any other inputs. Therefore, an important question is how to obtain the emergence of interactive capabilities with any architecture having a possible conflict between perceptive and predictive inputs? From the theoretical model described in section 2.1.3 the idea is to start from the connection of two systems simulating a robot-robot interaction (see Fig 6), and to exploit for free the dynamics of the interaction. Our working hypothesis is that the predictive layer plays the role of an endogenous oscillator, producing internal energy (the predictions) for the motor output, while the perceptive input plays the role of the modulation link allowing to add or subtract energy to the motor layer and favour a global synchrony or anti-synchrony of both agents. Once again, the solution is inspired by the C. Huyggens "entrainment" phenomenon: our system's perception is considered as an energy playing the same role than the phys- 
ical wave transmitted by the support in Huyggens experiment.

In the next sections, we will describe more precisely the details of the architecture , and the interaction experiment.

\subsubsection{Energy based architecture}

The Perception-Action architecture studied is a Neural network with the following properties (see the architecture Fig. (7):

A reflex pathway links actions (the Motor Output, MO) with perceptions (the Input group, Input). Perception is processed by the Input Group and sent to $M O$. If the resulting $M O$ potential overcome a given Motor threshold it triggers a response (an action) from the $M O$ neuron.

The output of $M O$ neurons is computed as follows:

$$
\begin{aligned}
& \operatorname{Pot}_{i}^{M O}=\sum_{j}\left(W_{i, j}^{\text {Input }} \cdot A c t_{i, j}^{\text {Input }}\right) \\
& A c t_{i, j}^{M O}=f_{M O}\left(\text { Pot }_{i, j}^{M O}\right) \\
& f_{M O}(x(t))= \begin{cases}1 & \text { if } x(t)>\theta_{M O} \\
0 & \text { otherwise }\end{cases}
\end{aligned}
$$

This direct Input-MO connection, ensures simple reflex behaviors at the basis of sensori-motor exploration and task learning 1644 . In order to exploit this emerging "spontaneous" imitative behavior, our architecture is composed of a second pathway connecting a Transition Learning and Prediction (TLP) network. The role of this network is to allow the architecture to learn and predict the timing of the changing sensory-motor events. The TLP system is recording proprioceptive information, that is to say the successive activations of the $M O$ group: the system learns its own sequence of actions. The TLP is inspired by the functions of two brain structures involved in memory and timing learning: the cerebellum and the hippocampus (see [6] for the neurobiological model we have proposed). TLP is composed of three groups of neurons (Fig. (7): Time Derivation $(T D)$ group, Time Base $(T B)$ group and Prediction output $(P O)$ group. These three groups will learn and predict the timing of the transitions between MO neurons. The neurons of the Prediction (PO) group learn the association between incoming proprioceptions via $T D$, the present, and the time trace of the last event on $T B$ (the past). The connectivity plays an important role in the transition learning.

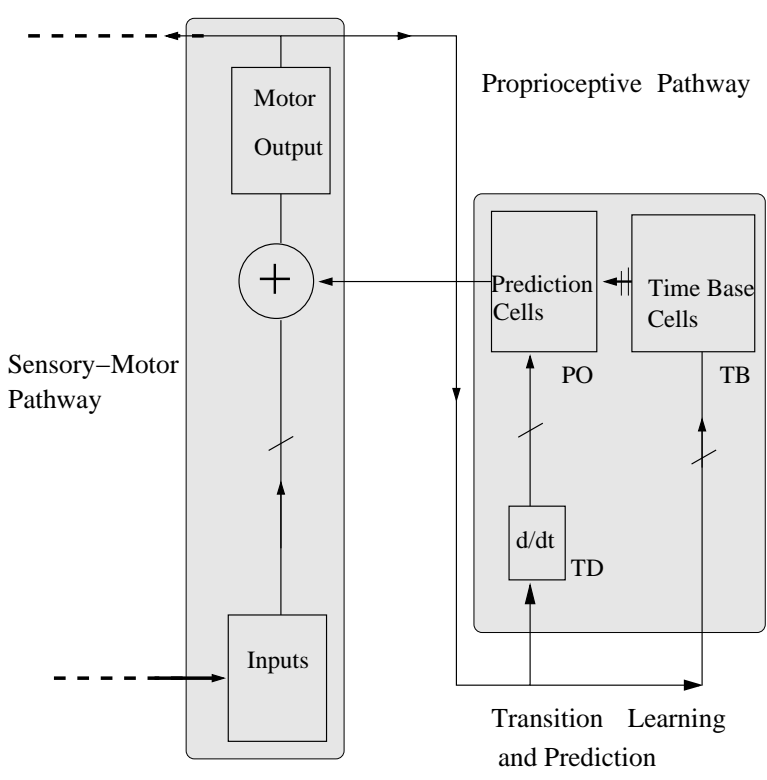

Fig. 7. Details of the Perception-Action architecture. The Input-Motor Output link represents a "reflex" pathway. A second loop pathway allow a Transition Learning and Prediction group to learn the sensori-motor flow under the form of a sequence of sensori-motor transition. As a result, after a learning phase and TLP is able to recall step by step the transition and to replay the learned sequence.

To each neuron in $M O$ corresponds a detector in $T D$ (a simple derivator to detect new events), and to each neuron of $M O$ corresponds one battery of $T B$ neurons firing with different time constants depending of $j$ :

$\operatorname{Act}_{j, l}^{T B}(t)=\left\{\begin{array}{lr}\frac{n \cdot \Theta}{T_{\max } \cdot(j+1)} \cdot t & \text { if } t<T_{\max } \\ \theta_{T B} & \text { otherwise }\end{array}\right.$

The $P O$ activity is computed classically, depending on the output of $T B$.

$$
\begin{aligned}
& \operatorname{Pot}_{i, j}^{P O}=\sum_{l} W_{p o(i, j)}^{T B(j, l)} \cdot A c t_{j, l}^{T B} \\
& A c t_{i, j}^{P O}=f_{P O}\left(\operatorname{Pot}_{i, j}^{P O}\right) \\
& f_{P O}=\exp \frac{x^{2}}{2 \cdot \sigma^{2}}
\end{aligned}
$$

Because of the connectivity between $T D, T B$ and $P O$, each $P O$ neuron can learn a given transition between two events. To $n$ possible events corresponds $n \times n$ neurons in $P O$. The potential of a $P O$ neuron is the sum of the information coming from $T D$ and the delayed activity in $T B$. The $(i, j)$ neuron learns the transition between the $i^{\text {th }}$ neuron of $T D$ 
and the $j^{\text {th }}$ group of $T B$ (itself corresponding to the time trace of the $j^{\text {th }}$ neuron of $M O$ ). Finally, merging the activities coming from $P O$ and Input to activate $M O$ plays a crucial role. The overshot of $\theta_{M O}$ by $P O$ or Input will trigger the action execution (Eq. 16). Figure 8 gives a summary of the different cases of functioning:

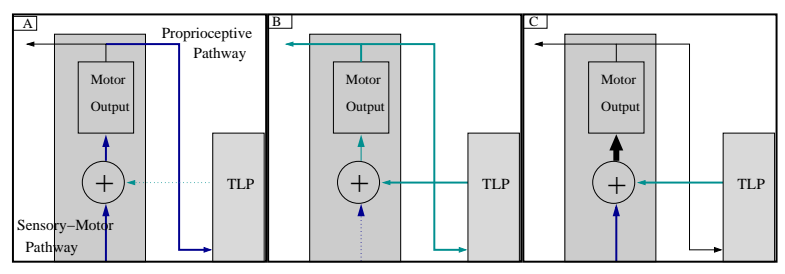

Fig. 8. Importance of the information flow in the functioning of the architecture. A) During learning, Input information drives the system, triggers $M O$ and the learning of the transition by $T L P$ B) When a sequence is learned, TLP and $M O$ (via the "proprioceptive" feedback) entertain their activities resulting in the recall of the sequence. C) During an interaction Input and $T L P$ are active, resulting in a potential increase of energy sent to $M O$, if $P O$ and Input are the same. This add of energy will result in an acceleration of the played sequence.

- During learning, the activity of the Input is important. Therefore, the Input-MO links are set to 1 , and an activity of Input directly triggers the corresponding $M O$ neuron (overshooting $\theta_{M O}$ ), in order to activate the $T L P$ groups and the learning of the sequence.

- When the system has learned a sequence, PO starts to fire and predict the timing of the transitions of the sequence. In order to obtain th complete reproduction of the sequence, each $P O$ response must activate the corresponding $M O$ neuron in order to generate the next transition prediction in $T L P$. Consequently, the $P O-M O$ links are set to 1 .

- Nevertheless, if the Input-MO link is critical for the learning process, it can as much interfere with the reproduction of a given sequence, as play a crucial role in the synchronisation process. During the experiments, the Input-MO links are set to values belonging to $[0,1[$. A sole activity from Input is not sufficient to overshot $\theta_{M O}$, but the co-activation of Input with a rising potential of the same $P O$ neuron induce an earlier overshot of $\theta_{M O}$ (because of the summation of Input and $P O$ activities on the same $M O$ neuron): in other words the system accelerates the triggering of $M O$, and repetitive co-occurrences will cause the system to synchronize $M O$ outputs on the perceived Input activities.

It is important to mention that during all the experiments, the weights of the $P O-M O$ links are set to 1 , while the weights of Input-MO where manually set to 1 during learning and $[0-1$ [ with a step of 0.1 during the different tests.

\subsubsection{Learning a sequence}

During the learning phase, the successive inputs are given to the system, causing $T L P$ to learn the transitions between each activated $M O$ neurons. The transitions correspond to the instants of coactivations of TD and TB, and are learned on the $T B-P O$ links. The learning rule is an associative "Delta rule" :

$\Delta W_{P O(i, j)}^{T B(j, l)}=\varepsilon \cdot\left(A c t_{j, l}^{T B}-W_{P O(i, j)}^{T B(j, l))}\right)$

Each $W_{P O(i, j)}^{T B(j, l)}$ encodes the activity level of $A c t_{j, l}^{T B}$ at the instant of the co-activation, and the $l$ links between the $l$ neurons of the $j^{\text {th }}$ group of $T B$ and the $P O$ neuron at position $(i, j)$ code the exact timing of the $i$ - $j$ transition. To recall a transition, the activation of the $i^{t h} M O$ neuron will cause the exact instant of the transition between $i$ and $j$ to be predicted when the $A c t_{i, j}^{P O}$ reaches its maximum value, corresponding to 1 . In order to reproduce the sequence, we trigger the system by stimulating the input of the first element of the sequence. This element will cause the prediction of the first transition, and in turn triggers the second element of the sequence, and so on. As a result the step by step predictions connected to the $M O$ group drive the recall and the reproduction of the sequence. Fig 9 details the learning and recall of a single transition AB.

\subsubsection{Experiment and results}

The output $(M O)$ of the first system is connected to the input (Input) of the second one, and conversely. The experiment was conducted as follow : first, the two agents learned apart the same sequence composed of the activation of three different inputs : A, then B then C, then A. Each activation simulates the recognition of a different sensori-motor category by the system.

After the learning phase, each agent is then able to reproduce the sequence. For each agent, a sole stimulation of the first element of the sequence (here A) starts the TLP predictions and the step by step recall of the whole sequence at the learned timing. In all the experiments, the first agent, $\left(\right.$ Agent $\left._{1}\right)$ was 

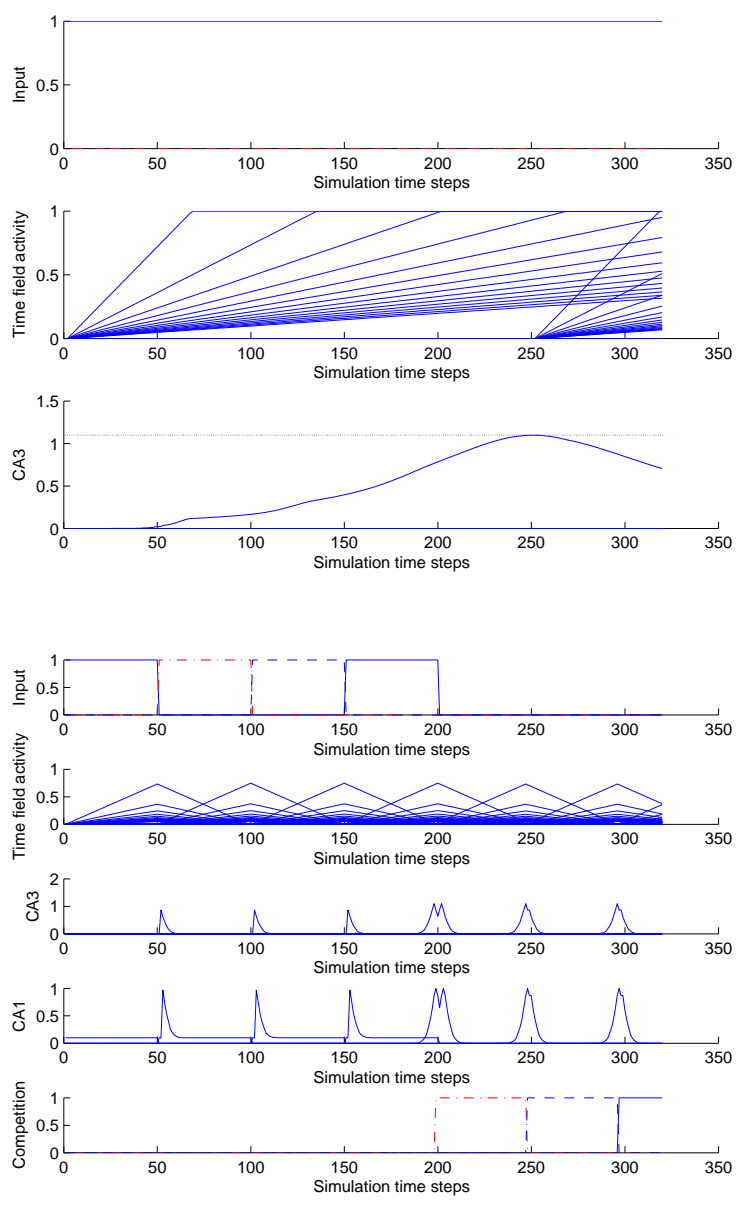

Fig. 9. Up : Example of a prediction of a transition on a $P O$ neuron. activities of Input, Time Base (TB), and Prediction $(P O)$ neurons. Before this record, the system was stimulated with input A from time step 0 to 250 , and with input B at time step 250. This figure shows activities during the recall of the $P O_{(i, j)}$ neurons whose activity reach its maximum at time step 250. Bottom: Learning and recalling a typical sequence used in our experiments. A sequence of 4 items activating 3 different neurons of Input group is presented to the system ( ABCA, at time steps 0 to 200). Once the sequence is learned, at time step 200 the $T L P$ starts to predict and reproduce the sequence.

taught the same reference sequence at a period $t_{1}=$ 10 seconds, meaning that each element of the sequence is lasting 10 seconds. In order to study the dynamics of the interaction, and especially phase locking of two agents, the period of the second agent (Agent2) was taught with different values going from $t_{2}=1$ to $t_{2}=30$. For each $t_{2}$, we tested eleven different cases of interactions corresponding to the modification in both agents of the values of the Input - MO link with values belonging to $[0.0,1.0]$ with steps of 0.1 . A value of 0.0 indicates that the Inputs have no effect on the system acceleration, and that the agents are not able to modulate the timing of their actions. Conversely, a value of 1.0 indicates that Input is able solely to overshoot $\theta_{M O}$ and triggers the system's action without taking into account the predictions. In the range ]0.0,0.1[ the acceleration of both systems happens with a strength depending on the instant of the co-occurrence of $P O$ and Input. Each control architecture was running on a separate Linux workstation, and exchanged information via the standard 100MB local network of the laboratory. It is important to mention that during the experiments, there where no particular attention or will to isolate the computers or the sub-network from the Lab. It means that on each workstation, the control architecture was running with the same priority than other processes (potentially cpu consumers) and that the delay of transmission of the information from $M O$ of an agent to Input of the other one could be uncertain. These experimental conditions can be seen as equivalent to those of future experiment on real robots (for example in a face to face setup) where interferences can come from the internal or the external environment. Figure 10

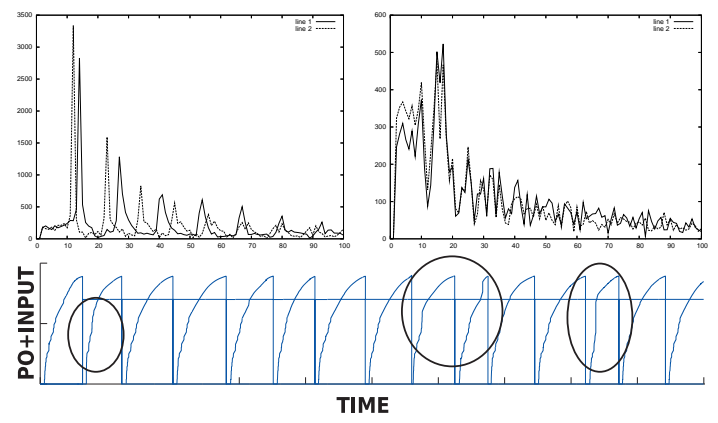

Fig. 10. Left: FFT transform of both agents $M O$ output. The interaction weight Input-MO is set to 0.0 There is thus no synchronization, and each agent plays the sequence at its original frequency $\left(t_{1}=10\right.$ vs $\left.t_{2}=13\right)$. Right: Same experiment $\left(t_{1}=10\right.$ vs $\left.t_{2}=13\right)$ with Input-MO set to 0.8 . Both agents synchronize. Bottom: Detailed activity of the fusion of $P O$ and Input of Agent $_{1}$ during the interaction corresponding to experiment b). A Continuous modulation can be seen, with important catch up can be seen at iterations $0.2,1.3$ and 1.7

shows the FFT of $P o t^{M O}$ of both systems during the two experiments. For all experiments, a Input-MO link with a weight of 0.0 results in no synchronization, each system playing its own sequence with its 
own initial period $t$. For the eleven experiments conducted with a period $t_{2}=1$, (whatever the value of Input-MO is) there was also no global synchronization, due to the too strong difference between $t_{1}=$ 10 and $t_{2}=1$. For the experiments conducted with $t_{2}$ going from 2 to 30 , we can observe a progressive and strong synchronization of the motor production of both systems. Perception thus results in an earlier triggering of the chosen action. To sum up, when the agents are connected together, the effect of producing cyclically the same sequence with a positive coupling results in a step by step synchronization of both behaviors, due to mutual accelerations when prediction and perception coincide.

\section{Discussion}

From an initial theoretical neural model, abstracting an interactive agent as a simple oscillating perception-action system we have shown that :

(i) the formal coupling of two of these NN oscillators leads to two different dynamics: a positive coupling involving synchrony and a negative coupling involving phase opposition,

(ii) the computational simulation of this coupling confirmed these dynamics with coupling parameters belonging to a large range of values,

(iii) the application of the negative coupling in the frame of a robotic demonstrator leads to a stable interaction, where each robot alternates active and passive behaviors, and where both actors take turn,

(iv) the application of the positive coupling in the frame of a simulated, real time, interaction between two embedded control architectures leads to a stable interaction, where both systems are able to synchronize when demonstrating the same sequence.

It is important to notice that in the case of a negative coupling, the turn taking is an emergent behavior. It should facilitate the design of intuitive human-robot interactions, but also robot-robot interactions. Indeed, we can imagine that different systems, with different morphologies or simply with different motor repertories should be able to alternate their turns of activity under the condition that they embed this oscillating motivation for interaction. This could provide a basic, but robust interaction which should favor the exchanges and the sharing of each agent's motor repertoire. In the frame of a positive coupling, it is interesting to notice that the synchrony between the two agents is also an emergent behavior, under the important condition that both agents are doing the same thing, i.e initially share some parts of their motor repertory. In this case, both agents synchronize their production with noticeable adjustments of the timing of their motor production. Therefore, in a situation of synchronization, the dynamics of an agent is slightly different from the one occurring when reproducing solely a sequence. The synchronization state could be easily characterizable and then could allow the systems to detect that it is interacting with "something" doing "the same thing at the same time". In conjunction with immediate imitation (allowing to "kick off" gestural games), we believe that synchrony is a good means to detect if the others can interact, in order to reach important stable states that may be involved in the recognition of the behaviors of others. As a characterizable "marker" of the interaction, synchrony can in turn be exploited as a transition state for behavior selection or modulation. It should help autonomous systems to stay locked, alternate roles, select and enrich their motor repertory via interaction, in a word to communicate.

For the moment, we have considered the 2 architectures as separated: one devoted to phase opposition, the other to synchrony and learning. However, when observing imitation sessions between young infants, "turn-taking" and synchrony are tightly imbricated [37]: in experiments involving pairs of child sharing similar objects, sequences of reciprocal imitation are observed. During a given period, one children takes turn and demonstrate something. The other tries to reproduce in synchrony, mainly for the pleasure of communication. Then they could eventually alternate their role. Regarding this experiment, it must be noticed that "turn-taking" and "synchrony" cannot be described at the same timescale: synchrony occurs within a "turn". In consequence, "turn-taking" can be considered as a phenomenon occurring at a higher timescale, having a low frequency. Conversely, synchrony must be analysed at a micro-level during the interaction of the two children. Coming back to our model, it would suggest that the oscillator regulating the "turn-taking" mechanism should modulate the learning and reproduction of sequences of action. It could act as a motivation for learning or being in synchrony. We are now exploring this idea unifying our 2 architectures. 


\section{Acknowledgements}

This work was supported by the European project Feelix Growing FP6 IST-045169

\section{References}

[1] P. Andry, P.Gaussier, and J. Nadel. Autonomous learning and reproduction of complex sequences: a multimodal architecture for bootstraping imitation games. In Fifth international workshop on epigenetic robotics, EPIROB 05, pages 97-101, 2005.

[2] V.I. Arnold. Remarks on the perturbation problem for problems of mathieu type. Math. Surveys, (38):215-233, 1983.

[3] J. Aschoff. A companion to animal physiology., chapter The circadianrhythm of body temperature as a function of body size, pages 173-188. Cambridge Univ. Press, Cambridge, 1982.

[4] V. Babeau, P. Gaussier, C. Joulain, A. Revel, and J.P. Banquet. Merging visual place recognition and path integration for cognitive map learning. In The Sixth International Conference on the Simulation of Adaptive Behaviour, SAB 2000, Paris, september, 2000.

[5] Jean-Paul Banquet, Philippe Gaussier, Jean-Claude Dreher, Cédric Joulain, and Arnaud Revel. Spacetime, order and hierarchy in fronto-hippocamal system : A neural basis of personality. In G. Mattews, editor, Cognitive Science Perspectives on Personality and Emotion, pages 123-189. Elsevier Science BV Amsterdam, 1997.

[6] J.P. Banquet, P. Gaussier, J.C. Dreher, C. Joulain, and A. Revel. Cognitive Science Perspectives on Personality and Emotion, chapter Space-Time, Order and Hierarchy in Fronto-Hippocampal System: A Neural Basis of Personality, pages 123-189. Elsevier Science BV Amsterdam, 1997.

[7] M. S. Baptista and J. Kurths. Chaotic channel. Physical Review E (Statistical, Nonlinear, and Soft Matter Physics), 72(4):045202, 2005.

[8] R.D. Beer. On the dynamics of small continuoustime recurrent networks. Technical Report CES-94-18, Cleveland, OH, 1994.

[9] F. Bernieri and J.S. Reznickand R. Rosenthal. Synchrony, pseudo synchrony, and dissynchrony: Measuring the entrainment process in mother-infant interactions. Journal of Personality and Social Psychology, 54(2):243-253, 1988.

[10] L. Berthouze and M. Lungarella. Motor skill acquisition under environmental perturbation: On the necessity of alternate freezing and freeing degrees of freedom. Adaptive behavior, 12(1):47-64, 2004.

[11] C. Breazeal. Regulation and entrainment for humanrobot interaction. International Journal of Experimental Robotics, 10-11(21):883-902, 2003.

[12] G. Butterworth. Imitation in Infancy, chapter Neonatal imitation: existence, mechanisms and motives, pages 6388. Cambridge University Press, 1999.
[13] G. Cheng and Y. Kuniyoshi. Complex continuous meaningful humanoid interaction: A multi sensory-cue based approach. In Proceedings of IEEE International Conference on Robotics and Automation (ICRA2000), pages 2235-2242, April 2000.

[14] N. Cuperlier, M. Quoy, and Ph. Gaussier. Neurobiologically inspired mobile robot navigation and planning. Frontiers in NeuroRobotics, 1(1), 2007.

[15] R. Elbe and W. Koller. Tremor. Johns Hopkins University Press, Baltimore., 1990.

[16] P. Gaussier and S. Zrehen. Complex architectures for autonomous agents. In P. Gaussier and J.D. Nicoud, editors, PerAc, pages 278-290, Lausanne, 1994. IEEE Press.

[17] Philippe Gaussier, Sorin Moga, Jean-Paul Banquet, and Mathias Quoy. From perception-action loops to imitation processes. Applied Artificial Intelligence (AAI), 1(7):701-727, 1998.

[18] C. Giovannangeli and Ph. Gaussier. Human-robot interactions as a cognitive catalyst for the learning of behavioral attractors. In 16th IEEE on Robot and Human Interactive Communication 2007, pages 10281033, 2007.

[19] C. Giovannangeli and Ph. Gaussier. Orientation system in robots: Merging allothetic and idiothetic estimations. In 13th International Conference on Advanced Robotics (ICAR07), pages 349-354, 2007.

[20] C. M. Gray, P. Konig, A. K. Engel, and W. Singer. Oscillatory responses in cat visual cortex exhibit intercolumnar synchronization which reflects global stimulus properties. Nature, (338):334-337, 1989.

[21] E. Basar H. Flohr H Haken and A. J. Mandell. Synergetics of the brain. New York: Springer-Verlag, 1993.

[22] H. Haken. Information and self-organization: $A$ macroscopic approach to complex systems. SpringerVerlag., London, 1988.

[23] C. Huygens. Instructions concerning the use of pendulum-watches for finding the longitude at sea. Phil. Trans. R. Soc. Lond., 4:937, 1669.

[24] A.J. Ijspeert, J. Nakanishi, and S. Schaal. Movement imitation with nonlinear dynamical systems in humanoid robots. In Proceedings of the IEEE International Conference on Robotics and Automation (ICRA2002), pages 1398-1403, 2002. (received the ICRA2002 best paper award).

[25] Auke Jan Ijspeert. A neuromechanical investigation of salamander locomotion. In Proceedings of the International Symposium on Adaptive Motion of Animals and Machines (AMAM 2000), 2000.

[26] J. Issartel and L. Marin M. Cadopi. Unintended interpersonal co-ordination: "can we march to the beat of our own drum?". Neuroscience Letters, 411:174-179, 2007.

[27] H. Kozima, C. Nakagawa, and H. Yano. Can a robot empathize with people? Artificial Life and Robotics, 8(1):83-88, September 2004.

[28] K.Prepin and A.Revel. Human-machine interaction as a model of machine-machine interaction: how to make machines interact as humans do. Advanced Robotics, 21(15):1709-1723, 2007.

[29] G. Kugiumutzakis. Imitation in Infancy, chapter Genesis and development of early infant mimesis to 
facial and vocal models, pages 36-59. Cambridge University Press, 1999.

[30] Matthieu Lagarde, Pierre Andry, and Philippe Gaussier. The role of internal oscillators for the one-shot learning of complex temporal sequences. In ICANN 2007, volume 4668 of $L N C S$, pages 934-943, 2007.

[31] W.A. MacKay. Synchronized neural oscillations and their role in motor processes. Trends in Cog.Sci., 1:176$183,1997$.

[32] O. Maratos. The origin and development of imitation in the first sixth month of life. Paper presented at the British Psychological Society Annual Meeting, Liverpool, April 1973.

[33] M. K Meltzoff, N. Moore. Imitation of facial and manual gestures by humans neonates. Science, 198:75-82, 1977.

[34] M.Masahiro. On the uncanny valley. In Proceedings of the Humanoids-2005 workshop: Views of the Uncanny Valley., Tsukuba, Japan., December 2005.

[35] RY. Moore. Circadian rhythms: A clock for the ages. Science, 5423(284):2102-2103, 1999.

[36] J. Nadel. Imitation et communication entre jeunes enfants. Presse Universitaire de France, Paris, 1986.

[37] J. Nadel and A. Peze. New Perspectives in Early Communicative Development. Routledge, London and New York, 1993.

[38] Z. Neda, E. Ravasz, Y. Brechet, T. Vicsek, and A.L. Barabasi. The sound of many hands clapping: Tumultuous applause can transform itself into waves of synchronized clapping. Nature, 403(6772):849-850, 2000.

[39] G. Nicolis and I. Prigogine. Self Organization in NonEquilibrium Systems, chapter Chaps. III and IV. J. Wiley and Sons, New York, 1977.

[40] K. Niizeki, K. Kawahara, and Y. Miyamoto. Interaction among cardiac, respiratory, and locomotor rhythms during cardiolocomotor synchronization. Journal of Applied Physiology, 75(4):1815-1821, 1993.

[41] O. Oullier, G.C. de Guzman, K.J. Jantzen, J. Lagarde, and J.A.S. Kelso. Social coordination dynamics: Measuring human bonding. Social Neuroscience, in press, 2008.

[42] Louis M. Pecora and Thomas L. Carroll. Synchronization in chaotic systems. Phys. Rev. Lett., 64(8):821-824, Feb 1990.

[43] JC. Peery. Neonate-adult head movement - no and yes revisited. Developmental Psychology., pages 245-250, 1980.

[44] Rolf Pfeifer and Christian Scheier. Understanding Intelligence. The MIT Press, 2001.

[45] A. Pikovsky, M. Rosenblum, and J. Kurths. Synchronization: A Universal Concept in Nonlinear Sciences. Cambridge University Press, 2001.

[46] B. Van Der Pol. On relaxation oscillation. Phil. Mag., 2:978-992, 1926.

[47] F. Poyatos. New Perspectives on Nonverbal Communication. Pergamon Press, Oxford, 1983.

[48] P. Sassone-Corsi. Molecular clocks : mastering time by gene regulation. Nature, 362:871-874, 1998.

[49] W. Singer. Striving for coherence. Nature, 397(6718):391-393, 1999.

[50] P. N. Steinmetz, A. Roy, P. Fitzgerald, S.S. Hsiao, E. Niebur, and K. O. Johnson. Attention modulates synchronized neuronal firing in primate somatosensory cortex. Nature, (404):187-190, 2000.

[51] M. Stopfer, S. Bhagavan, B.H Smith, and G. Laurent. Impaired odour discrimination on desynchronization of odour-encoding neural assemblies. Nature, 6(390):7074, 1997.

[52] R.L. Stratonovich. Topics in the Theory of Random Noise. Gordon and Breach, New York, 1963.

[53] S. H. Strogatz. Sync: The emerging science of spontaneous order. Hyperion Press, 2003.

[54] P. Tass, J. Kurths, M. Rosenblum, J. Weule, A. Pikovsky, J. Volkmann, H. Schnitzler, and H. Freund. Analysis of Neurophysiological Brain Functioning, chapter Complex phase synchronization in neurophysiological data., pages 252-273. SpringerVerlag, Berlin, 1999.

[55] E. Tognoli, J. Lagarde, G. C. DeGuzman, and J. A. S. Kelso. The phi complex as a neuromarker of human social coordination. PNAS, 104(19):8190-8195, 2007.

[56] W.Singer and C.M.Gray. Visual feature integration and the temporal correlation hypothesis. Ann.Rev. Neurosci., 18:555-586, 1995. 